\title{
ON THE CHARACTERS OF UNITARY REPRESENTATIONS
}

\author{
MICHAEL COWLING
}

(Received 5 March 1987)

Communicated by J. F. Price Dedicated to Robert Edwards in recognition of
25 years' distinguished contribution to mathematics in Australia,
on the occasion of his retirement

\begin{abstract}
Let $G$ be a locally compact group, and let $D(G)$ be a dense subalgebra of the convolution algebra $L^{1}(G)$. Suppose that $\pi$ is a unitary representation of $G$ and that, for each $u$ in $D(G), \pi(u)$ is a trace-class operator. Then the linear functional $u \rightarrow \operatorname{tr}(\pi(u))$ (the trace of $\pi(u)$ ) is called the $D$-character of $\pi$. We give a simple proof that the $D$-character of such a representation determines the representation up to unitary equivalence. As an application, we give an easy proof of the result of Harish-Chandra that the $K$-finite characters of unitary representations of semisimple Lie groups determine the representations.
\end{abstract}

1980 Mathematics subject classification (Amer. Math. Soc.): 22 D 10, 22 D 25.

The point of this paper is ta insert into the literature simpler and more general proofs of some known results in representation theory, described in the abstract. J. Dixmier ([2], 17.2.1) showed that the $D$-character of a unitary representation determines the representation, but he required the extra hypothesis that $D(G)$ be closed under the involution of $L^{1}(G)$, and his proof is more complicated than ours: Harish-Chandra proved that $K$-finite unitary characters determine the unitary characters from which they arise, but, to quote J. A. Wolf ([5], page 122), the proof involves "a certain amount of machinery comparing Naimark equivalence, infinitesimal equivalence, Banach equivalence, and unitary equivalence". It seems worthwhile to point out that this important result has a proof

(C) 1988 Australian Mathematical Society $0263-6115 / 88 \$ A 2.00+0.00$ 
which relies only on elementary facts about unitary representations and compact operators.

We shall need a few standard facts about $C^{*}$-algebras associated to group representations. Let $G$ be a locally compact group, and $L^{1}(G)$ be the usual Lebesgue space constructed relative to a left-invariant Haar measure $d m$. By $\hat{G}$ we denote the set of all irreducible unitary representations $\pi$ of $G$ on Hilbert spaces $H_{\pi}$, modulo unitary equivalence. Such representations extend naturally to $L^{1}(G)$ by the rule

$$
\pi(u)=\int_{G} d m(g) u(g) \pi(g) \quad \forall u \in L^{1}(G) ;
$$

$\|\pi(u)\|_{\text {op }}$ denotes the operator norm of $\pi(u)$. By $C^{*}(G)$ we mean the completion of $L^{1}(G)$ in the norm \|\|$_{*}$ :

$$
\|u\|_{*}=\sup \left\{\|\pi(u)\|_{\text {op }}: \pi \in \hat{G}\right\} \quad \forall u \in L^{1}(G) .
$$

Then $C^{*}(G)$ is a $C^{*}$-algebra, which is discussed by P. Eymard [3].

If $\sigma$ is a completely continuous representation of $G$ (that is, if $\sigma(u)$ is a compact operator on $\mathbf{H}_{\sigma}$ for each $u$ in $L^{1}(G)$ ), then $\sigma$ can be decomposed into a direct sum of irreducible unitary representations

$$
\sigma=\sum_{\pi \in P}^{\oplus} m_{\pi} \pi
$$

where $P$ is the subset of $\hat{G}$ containing all completely continuous unitary representations, and $m_{\pi} \in \mathbf{N}_{0}$ (see J. Dixmier [2], 5.4.13). Let $Q$ be the subset of $P$ of those representations $\pi$ for which $m_{\pi} \neq 0$. We write

$$
T=\sum_{\pi \in Q}^{\oplus} m_{\pi} T_{\pi}
$$

to indicate that $T$ is an operator on $\sum_{\pi \in Q}^{\oplus} m_{\pi} \mathbf{H}_{\pi}$ which acts by the operator $T_{\pi}$ on each of the $m_{\pi}$ copies of $\boldsymbol{H}_{\boldsymbol{\pi}}$ in the Hilbert space sum. Since

$$
\sup \left\{\|\pi(u)\|_{\text {op }}: \pi \in Q\right\} \leq\|u\|_{*} \quad \forall u \in L^{1}(G),
$$

we may extend $\sigma$ to $C^{*}(G)$. Then the image $\sigma\left(C^{*}(G)\right)$ is the $C^{*}$-algebra $c_{0}(Q ; \mathcal{L C})$, by which we mean the space of all operators $\sum_{\pi \in Q}^{\oplus} m_{\pi} T_{\pi}$ on $\sum_{\pi \in Q}^{\oplus} m_{\pi} H_{\pi}$ such that each $T_{\pi}$ is compact and such that $\pi \rightarrow\left\|T_{\pi}\right\|_{\text {op }}$ is in $c_{0}(Q)$. This result follows from the observation that $\sigma(u)$ is in $c_{0}(Q ; \mathcal{L C})$ because it is compact, together with the observation that, since $\sigma$ is a *-morphism of $C^{*}(G)$ to a dense subalgebra of $c_{0}(Q ; \mathcal{L C})$ which does not increase norms, then the quotient algebra $C^{*}(G) / \operatorname{ker}(\sigma)$ is isometrically isomorphic to $c_{0}(Q ; \mathcal{L} C$ ) (see Dixmier [2], 1.8.3). 
Let $D(G)$ be a subspace of $C^{*}(G)$ mapped by $\sigma$ into the space of trace class operators in $c_{0}(Q ; \mathcal{L C})$. Then the $D$-character of $\sigma$ is the linear functional $\chi_{\sigma}$ on $D(G)$ described by the formula

$$
\chi_{\sigma}(t)=\operatorname{tr}(\sigma(t)) \quad \forall t \in D(G) .
$$

The principal question of this paper is whether $\chi_{\sigma}$ determines $\sigma$, up to unitary equivalence. Obviously, $D$ needs to be large for $\chi_{\sigma}$ to determine $\sigma$, so it is reasonable to suppose that $D(G)$ is dense in $C^{*}(G)$, but this is not enough. For instance, if $\sigma$ is the regular representation of a compact Lie group and $D(G)$ is the subset of $C^{\infty}(G)$ of functions which vanish at the identity of $G$, then the $D$-character of $\sigma$ is identically zero. The extra hypothesis we use is simple and natural: it suffices to assume that $D(G)$ is closed under convolution (written *).

THEOREM 1. Suppose that $G$ is a locally compact group, and that $D(G)$ is a dense subalgebra of $L^{1}(G)$. If $\sigma$ is a unitary representation of $G$ and $\sigma(u)$ is a trace class operator for any $u$ in $D(G)$, then the $D$-character of $\sigma$ determines $\sigma$ up to unitary equivalence.

Proof. As above, we may write $\sigma=\sum_{\pi \in Q}^{\oplus} m_{\pi} \pi$, where $m_{\pi} \in \mathbf{N}$. Let $R$ be the set of all irreducible unitary representations of $G$ (or rather, equivalence classes thereof) which map $D(G)$ to the trace class. If $\pi \in \hat{G} \backslash R$, then $m_{\pi}=0$, so it suffices to determine $m_{\pi}$ for $\pi$ in $R$. Fix $\nu$ in $R$. We shall determine $m_{\nu}$.

Temporarily let $\tau$ denote the representation $\nu \oplus \sigma$, which can be written as a direct sum:

$$
\tau=\sum_{\pi \in Q}^{\oplus} n_{\pi} \pi,
$$

where $n_{\pi}=m_{\pi}$ if $\pi \neq \nu$ and $n_{\nu}=m_{\nu}+1$. Take $w$ in $D(G)$ so that $\nu(w)$ is non-trivial. By our introductory remarks, applied to $\tau$ rather than $\sigma$ if $m_{\nu}=0$, there exists $u$ in $C^{*}(G)$ so that $\pi(u)=0$ for all $\pi$ in $R \backslash\{\nu\}$ while $\nu(u)=\nu(w)^{*}$ (the adjoint of $\nu(w)$ ).

Since $D(G)$ is dense in $L^{1}(G)$ and hence in $C^{*}(G)$, there is a sequence $\left(u_{n}\right)$ of elements of $D(G)$ such that $\lim _{n \rightarrow \infty}\left\|u-u_{n}\right\|_{*}=0$. Now

$$
\lim _{n \rightarrow \infty} \operatorname{tr}\left|\sigma\left(\left(u-u_{n}\right) * w\right)\right| \leq \lim _{n \rightarrow \infty}\left\|u-u_{n}\right\|_{*} \operatorname{tr}|\sigma(w)|=0
$$

so that

$$
m_{\nu} \operatorname{tr}(\nu(u * w))=\operatorname{tr}(\sigma(u * w))=\lim _{n \rightarrow \infty} \operatorname{tr}\left(\sigma\left(u_{n} * w\right)\right),
$$

while similarly $\operatorname{tr}(\nu(u * w))=\lim _{n \rightarrow \infty} \operatorname{tr}\left(\nu\left(u_{n} * w\right)\right) \neq 0$. Thus $m_{\nu}$ is determined by the formula

$$
m_{\nu}=\lim _{n \rightarrow \infty} \chi_{\sigma}\left(u_{n} * w\right) / \lim _{n \rightarrow \infty} \chi_{\nu}\left(u_{n} * w\right)
$$


COROLlARY 2 (Harish-Chandra, see G. Warner [4, pages 342-343]). Suppose that $G$ is a connected semisimple Lie group and that $\tau$ and $\tau^{\prime}$ are $K$-finite unitary representations whose $K$-finite characters (Warner uses the appellation "characters") coincide. Then $\tau$ and $\tau^{\prime}$ are unitarily equivalent.

Proof. The space of smooth, compactly-supported, left and right $K$-finite functions on $G$ (Warner uses the notation $C_{*}^{\infty}(G)$ ) is a dense subalgebra of $L^{1}(G)$, and the theorem applies.

REMARK. The spaces $C_{c}^{*}(G)$ on a Lie group $G$, the space of locally constant compactly supported functions on a totally disconnected group, and the space $A_{c}(G)$ (see [1]) on any locally compact group are all examples of dense subalgebras of $L^{1}(G)$ on which characters may be naturally defined.

\section{References}

[1] M. Cowling, 'Some applications of Grothendieck's theory of topological tensor products in harmonic analysis', Math Ann. 232 (1978), 273-285.

[2] J. Dixmier, $C^{*}$-algebras (North-Holland Publishing Co., Amsterdam, New York, Oxford, 1977).

[3] P. Eymard, 'L'algèbre de Fourier d'un groupe localement compact', Bull. Soc. Math. France 92 (1964), 181-236.

[4] G. Warner, Harmonic analysis on semi-simple Lie groups. I (Springer-Verlag, Berlin, Heidelberg, New York, 1972).

[5] J. A. Wolf, M. Cahen and M. DeWilde (eds), Harmoric analysis and representations of semisimple Lie groups (D. Reidel Publishing Company, Dordrecht, Boston, London, 1980).

School of Mathematics

University of New South Wales

P. O. Box 1

Kensington, N.S.W. 2033

Australia 